\title{
Samarium Metal-Induced Reactions: Synthesis of Diverse Organic Compounds
}

\section{Bimal Krishna Banik}

Community Health Systems of South Texas, Edinburg, Texas 78539, USA

"Corresponding author: Bimal Krishna Banik, Community Health Systems of South Texas, Edinburg, Texas 78539, USA, Tel: 09566658741; E-mail: bimal.banik@chsst.org

Received date: November 24, 2017; Accepted date: November 30, 2017; Published date: December 06, 2007

Copyright: (c) 2017 Banik BK. This is an open-access article distributed under the terms of the Creative Commons Attribution License, which permits unrestricted use, distribution, and reproduction in any medium, provided the original author and source are credited.

\section{Abstract}

Diverse reactions with samarium metal in the presence of different additives are studied. These methods produce useful compounds. Electron transfer mechanism is proposed.

Keywords: Samarium metal; Reduction; Reductive dimerization; Barbier reaction; Coupling

\section{Introduction}

Samarium metal is commercially available and economical. The use of this metal in different types of organic transformations in our laboratory is documented. Some of the resulting products serve as valuable intermediates for the synthesis of biologically active molecules.

\section{Results and Discussions}

\section{Reduction of the aromatic nitro groups}

Aromatic nitro groups are converted to aromatic amines using samarium metal and catalytic amounts of iodine [1]. Nitro groups in monocyclic to pentacyclic aromatic systems are reduced conveniently by samarium/iodine. For example, several benzene, naphthalene, anthracene, phenanthrene, pyrene, chrysene and dibenzofluorenes substituted with a nitro group in any location of these systems are reduced in excellent yields to their corresponding amines.

Ultrasound-induced samarium metal in the presence of ammonium chloride is a strong reagent combination in the reduction of aromatic nitro compounds to aromatic amines. The reaction proceeds at a very high speed. No intermediate formation of nitroso or hydroxylamine derivatives are observed in the above two reactions [2]. The success of this reaction can be rationalized by the formation of samarium iodide in the reaction media and this reduces the aromatic nitro groups. In reality, bivalent samarium iodide cannot reduce some of the aromatic nitro compounds used in this study. However, partial reduction of aromatic nitro groups to amines along with other intermediates is observed. In contrast of using molar proportion of iodine, catalytic amount of iodine is found to be necessary for such a reduction reaction. Therefore, the role of iodine is not clear in this reaction. The activation of samarium metal by iodine and subsequent series of electron transfers to the aromatic nitro groups is also possible.

The amino compounds obtained from these studies are converted to several analogues that have demonstrated anticancer activities [3-5].

\section{Reduction and reductive dimeization of imines}

Imines of diverse structures are dimerized to secondary amines using samarium metal in the presence of catalytic amounts of iodine [6]. This process involves single electron transfer to the $\mathrm{C}=\mathrm{N}$ bonds of the imines and subsequent radical-radical coupling process. Following this method, reductive dimerization of ketimines is also performed [7]. In some examples, considerable amount of monoamines are also formed. It depends on two competitive pathways.

Ferrocenyl imines produce only the monoamines under this reaction conditions. The iron containing imines on electron transfer by samarium produces an ion radical. This ion radical is unable to couple to each other due to the presence of iron-containing cyclopentadienyl system. Therefore, further electron transfer produced dianion which on protonation gives the monoamine as the only product [8].

\section{Reductive amination}

Imines are the products of a carbonyl compound and a primary amine. They can be used directly in a reaction without isolation because some imines are not stable. Amination of adamantyl system is performed by samarium-induced reductive amination procedure. Adamantyl ketone on reaction with primary amines produces imines. The intermediate imines on reduction with samarium in the presence of iodine give amino derivative of adamantyl system [9]. It appears, the bulky adamantyl ring prevents coupling reaction to occur.

\section{Reductive coupling of aromatic ketones}

Samarium metal in the presence of alkyl halides is a reagent that produces pinacol-types of coupling products of ketones. It is believed that alkyl halides react with samarium and produces reactive samarium halide species instead of alkyl samarium halide species. The reactive samarium halide specis then promotes radical-radical coupling and forms the dimeric compounds [10]. NBromosuccinimide-samarium is also useful for the reductive coupling of aryl ketones [11]. Ultrasound-induced method in the presence of ammonium chloride also produces the same compounds at a much faster rate [12]. A most probable mechanism of this process is advanced [13]. The rate of reaction in the presence of ammonium chloride is very fast. However, samarium in the presence of sodium chloride fails to produce any products. 
Citation: Banik BK (2017) Samarium Metal-Induced Reactions: Synthesis of Diverse Organic Compounds. Mod Chem Appl 5: 239. doi: $10.4172 / 2329-6798.1000239$

Page 2 of 2

\section{Barbier reaction of aromatic aldehydes and ketones}

After considerable experimentation, Barbier reaction of aromatic aldehydes and ketones becomes possible with samarium metal and alkyl halides [14].

\section{Reductive dimerization of cinnamate ester}

Reductive dimerization of methyl or ethyl cinnamate is realized with samarium metal in the presence of aluminum powder or aluminum chloride. This reaction is fascinating since all attempts to dimerize this type of unsaturated ester fail. The reduction of the unsaturated bond takes place in most of the instances. The presence of Lewis acid helps to stabilize the initially formed ion radical to undergo a coupling. As a result, dimer is formed. Finally, this dimer is converted to rare chrysene [15].

\section{Conclusions}

Samarium metal-induced reactions have produced a number of unknown compounds. However, this metal fails to reduce carboethoxy, amide, aromatic group, heteroaromatic ring, cyano and non-activated alkene groups. Usually these reactions did not proceed or complex products were formed. Since different additives were successfully used in combination with samarium metal for numerous reactions, it is expected that the chemistry of samarium metal can be extended in the near future. Studies can be included to identify chemoselective reduction and reductive dimerization. The coupling of unsaturated ester may open up the possibility of using other conjugated compounds. In conclusion, it is possible to obtain numerous compounds by conducting different types of reactions with samarium metal in the presence of suitable additives. It is important to note that samarium-induced reaction is fire-sensitive. Therefore, it is important that researchers take precautions when they perform samarium chemistry.

\section{Acknowledgment}

Bimal Krishna Banik is grateful to students and scientists who participated in these projects. He is also grateful to NIH, NCI, Kleberg Foundation, University of Texas M. D. Anderson Cancer Center, University of Texas-Pan American, University of Texas Health Science Center at San Antonio and Community Health System of South Texas.

\section{References}

1. Banik BK, Mukhopadhyay C, Venkatraman MS, Becker FF (1998) A Facile Reduction of Aromatic Nitro Compounds to Aromatic Amines by Samarium and Iodine. Tetrahedron Lett 39: 7243-7246.

2. Basu MK, Becker FF, Banik BK (2000) Ultra Sound-Promoted Samarium/ Ammonium Chloride Mediated Highly Efficient Reduction of Aromatic Nitro Compounds. Tetrahedron Lett 41: 5603-5606.

3. Becker FF, Banik BK (1998) Polycyclic Aromatic Compounds as Anticancer Agents: Synthesis and Biological Evaluation of Some Chrysene Derivatives. Bioorg Med Chem Lett 8: 2877-2880.

4. Becker FF, Mukhopadhyay C, Hackfeld L, Banik I, Banik BK (2000) Polycyclic Aromatic Compounds as Anticancer Agents: Synthesis and Biological Evaluation of Dibenzofluorene Derivatives. Bioorg Med Chem 8: 2693-2699.

5. Banik BK, Becker FF (2001) Polycyclic Aromatic Compounds as Anticancer Agents: Structure-Activity Relationships Study of New Chrysene and Pyrene Derivatives. Bioorg Med Chem 9: 593-605.

6. Banik BK, Zegrocka O, Banik I, Hackfeld L, Becker FF (1999) SamariumInduced Iodine-Catalyzed Reduction of the Imines: Synthesis of Secondary Amine Derivatives. Tetrahedron 40: 6731-6734.

7. Banik BK, Ghatak A, Samajdar S, Basu MK, Hackfeld L, et al. (2001) A New Synthetic Method for the Reduction of Imines by SamariumInduced Reaction. Indian J Chem 40B: 1134-1139.

8. Banik BK, Samajdar S, Ghatak A (2001) A Convenient SamariumMediated Reduction of Ferrocenyl Imines. Heterocycles 55: 1957-1961.

9. Banik BK, Zegrocka O, Becker FF (2000) Samarium-Mediated IodineCatalyzed Reductive Amination of Adamantyl Methyl Ketone. J Chem Res 2000: 321-323.

10. Ghatak A, Becker FF, Banik BB (2000) Samarium-Induced Reductive Coupling of Ketones in the Presence of Alkyl Halides. Tetrahedron Lett 41: 3793-3796.

11. Basu MK, Becker FF, Banik BK (2000) Ultrasound-Promoted Samarium/ Ammonium Chloride Mediated Reductive Coupling of Reduction of Aromatic Ketones. J Chem Res 2000: 406-407.

12. Banik BK (2003) Samarium-Induced Reductive Dimerization of Aryl Ketones in Aqueous Alcohol. Chem- Indian J 1: 149-150.

13. Banik I, Samajdar S, Cuellar R (2005) Samarium/NBS-Induced Reductive Dimerization of Carbonyl Compounds. Tetrahedron Lett 46: 2319-2322.

14. Basu MK, Banik BK (2001) Samarium-Mediated Barbier Reaction of Carbonyl Compounds. Tetrahedron Lett 42: 187-190.

15. Banik BK, Venkatraman MS, Banik I, Basu MK (2004) SamariumInduced Reductive Dimerization of Methyl Cinnamate: Synthesis of 2,8Diamino Chrysene. Tetrahedron Lett 45: 4737-4739. 